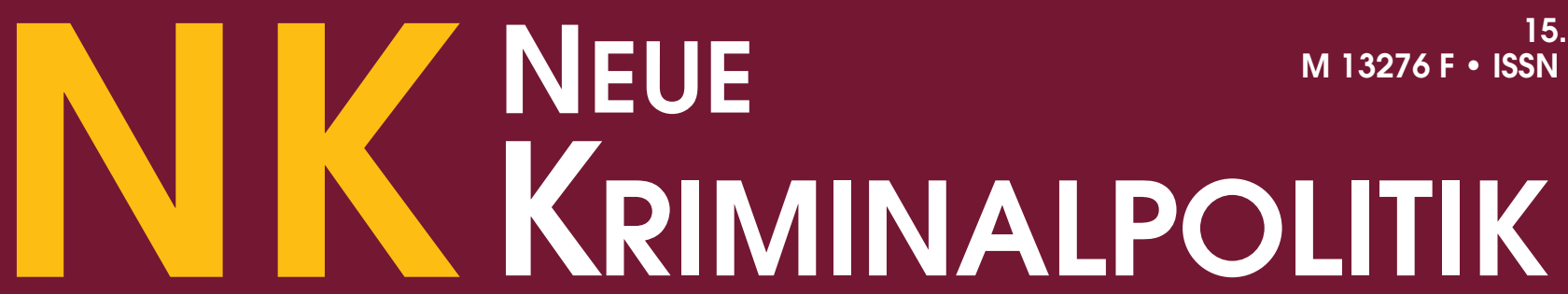

Forum für Praxis, Politik und Wissenschaft $\angle 03$

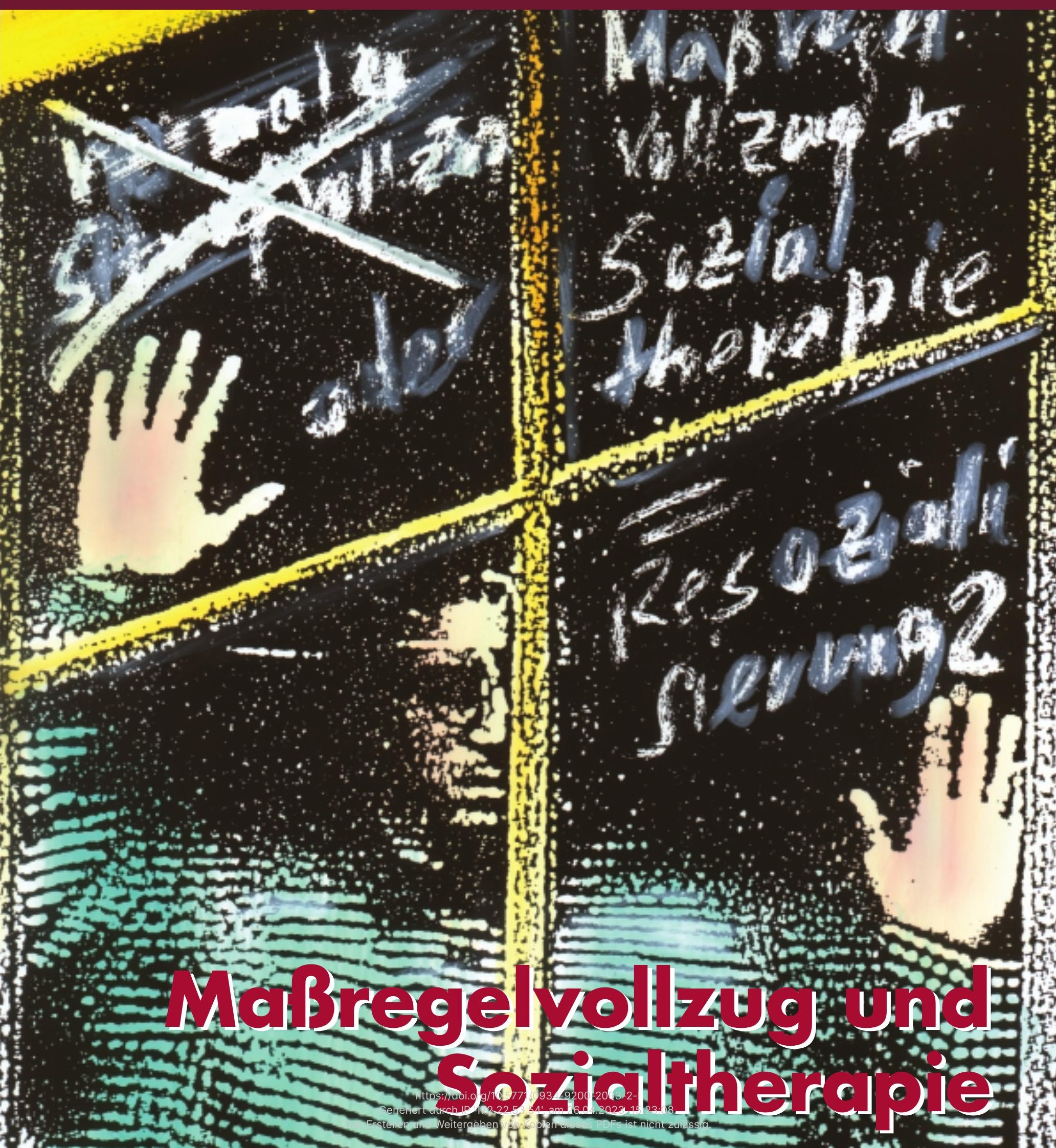




\section{NOMOS Aktuell}

\section{Das Deutsche Bundesrecht - Leitfäden}

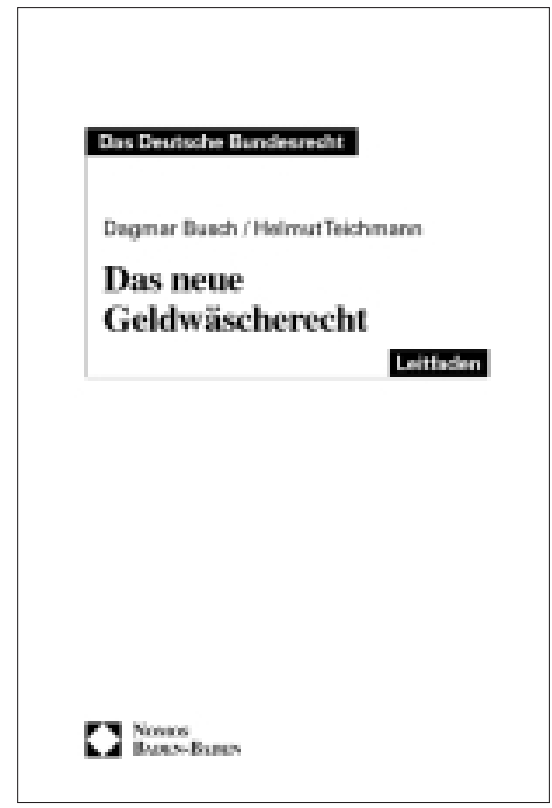

Dagmar Busch/Helmut Teichmann

\section{Das neve Geldwäscherecht}

2003, 125 S., brosch., 19,80 €, ISBN 3-7890-8 111 1-6

Das neue Geldwäschebekämpfungsgesetz ist am 15. August

2002 in Kraft getreten. Es hat sich zum Ziel gesetzt, eine

effektivere Bekämpfung von Geldwäsche und Finanzierung von internationalem Terrorismus zu ermöglichen. Die Darstellung der Novellierung hat u.a. folgende Schwerpunkte:

- Umsetzung der europäischen Geldwäsche-Richtlinie (Einbeziehung never Berufsgruppen wie z. B. Immobilienmakler, Rechtsanwälte, Notare, Steverberater, Wirtschaftsprüfer)

- Ausgestaltung einer deutschen Zentralstelle für Verdachtsanzeigen

- Umsetzung bisheriger Erfahrungen mit dem alten Geldwäschegesetz (z. B. verstärkte Berücksichtigung neuer Medien, Abbau bürokratischer Hemmnisse)

Die Autoren: Dr. Helmut Teichmann ist zuständiger Referatsleiter im Bundesministerium des Innern, Dagmar Busch seine Stellvertreterin. Beide waren federführend an Entstehung und Umsetzung des Geldwäschebekämpfungsgesetzes beteiligt.

\section{Marko Dörre}

\section{Der neue Jugendmedienschutz}

2003, 170 S., brosch., 24,80€, ISBN 3-7890-8312-7

Am 1. April 2003 ist mit dem In-Kraft-Treten des Jugendschutzgesetzes (JuSchG) und des Jugendmedienschutz-Staatsvertrages (JMStV) eine umfassende Neuregelung des Jugendmedienschutzes erfolgt. Unternehmen, Behörden, Anwälte und Gerichte müssen sich darauf einstellen.

\section{Achim-Volker König/Christian Papsthart}

\section{Das neue Waffenrecht}

\section{i. Vb., 2003, 200 S., brosch., 34,- €, ISBN 3-7890-8313-5}

Der Leitfaden »Waffenrecht« bietet eine umfassende Darstellung und Erläuterung des völlig neu konzipierten und inhaltlich geänderten Waffen- und Beschussrechts. Er ist für Anwender der Praxis wie für Interessierte generelle Grundlage für die Beantwortung waffenrechtlicher Fragestellungen. 
Helfen statt strafen

Auf diese kurze Formel kann man sich schnell einigen. So haben sich auch innerhalb des Strafsystems über die Jahre zahlreiche helfende Maßnahmen etabliert, von denen das vorliegende Heft einige aufgreift. Auf der einen Seite stehen Angebote wie die gemeinnützige Arbeit zur Vermeidung von Ersatzfreiheitsstrafen oder die Berufshilfe innerhalb und außerhalb des Strafvollzugs, bei denen das Bestreben, die negativen Folgen einer Freiheitsstrafe abzuwenden, klar im Vordergrund steht und die auf ein hohes Maß an Freiwilligkeit und Kooperation seitens der Betroffenen bauen. Auf der anderen Seite stehen Zwangsmaßnahmen wie der Maßregelvollzug und die Sozialtherapie, bei denen die Ambivalenz der Verkopplung von Hilfe und Strafe klar hervortritt. Hilfe mag zwar besser (gemeint) sein als Strafe, aber ihre Verwirklichung im Schatten des Strafrechts scheitert allzu oft an den widrigen Rahmenbedingungen. Unsere Autoren beschäftigen sich mit der konkreten Praxis in diesem schwierigen Feld, beleuchten verborgene Bereiche wie den Maßregelvollzug bei Jugendlichen und mit den Auswirkungen des neuen $\$ 9$ des Strafvollzugsgesetzes auf den Ausbau der sozialtherapeutischen Angebote und deren inhaltliche Ausgestaltung. Es zeigt sich, dass entgegen der öffentlichen Rede vom »Wegsperren « die Alternativen zum reinen Verwahrvollzug auf dem Vormarsch sind. Dennoch bleibt viel zu tun, die hochgesteckten gesetzlichen Ziele zu erfüllen und flächendeckende Angebote zu entwickeln, bei denen Hilfe nicht nur als Alibi herhalten muss. Im niedrigschwelligen Bereich der ambulanten Straffälligenhilfe spielen Freie Träger eine wichtige Rolle bei der Umsetzung alternativer Sanktionen. Doch hier fehlt es bislang an einer einheitlichen gesetzlichen Regelung.

Wie immer beste Fachlektüre wünscht Ihnen

Oliver Brüchert

\section{Titel: Maßregelvollzug und Sozialtherapie}

Zu einer »Maßregel der Besserung und Sicherung « wird man nicht verurteilt, sie wird vom Gericht angeordnet, wenn es zur Auffassung gelangt, es mit einem "gefährlichen Hangtäter zu tun zu haben. Voraussetzungen ist unter anderem das Vorliegen einer rechtswidrigen Straftat. Es folgt die Unterbringung in einem psychiatrischen Krankenhaus oder einer Entziehungsanstalt. Insbesondere für Sexualstraftäter sollen seit Anfang des Jahres spezielle sozialtherapeutische Anstalten zur Verfügung stehen. Die Titelbeiträge vermitteln einen Überblick über den aktuellen Zustand des Behandlungsvollzugs, über den gesetzlichen Rahmen, die Klientel und die Therapiekonzepte.

\section{MAGAZIN}

Ambulante Straffälligenhilfe:

Stärkung, Ausbau und Vernetzung

von Frieder Dünkel und Heinz Cornel

Österreich:

Führungswechsel und Statusaufnahme bei NEUSTART

von Arno Pilgram . . . . . . . . . . . . . . . . . . . . . .

Sexualstrafrecht:

Die Reform der Reform der Reform ...

von Monika Frommel . . . . . . . . . . . . . . . . . . . . . . .

Standpunkt:

Darf man foltern - wenigstens im Ausnahmefall?

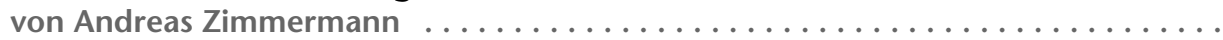

\section{THEMEN}

Rahmenbedingungen erfolgreicher Ableistung gemeinnütziger Arbeit Evaluationsergebnisse des Modells der Fachstellen in Nordrhein-Westfalen von Gabriele Kawamura-Reindl und Richard Reindl ...................

Berufshilfe und die Betreuung von Straffälligen

von Eduard Matt. ...............................

\section{TITEL}

Maßregelvollzug bei Jugendlichen in Deutschland

- erste Einblicke in eine verborgene Praxis

von Anne Tessenow und Heribert Ostendorf. . . . . . . . . . . . . . . . . . . . .

\section{Endlich Therapie für alle?}

Die Bundesländer und ihre sozialtherapeutischen Einrichtungen

von Kirstin Drenkhahn . . . . . . . . . . . . . . . . . . . . . . .

\section{Sozialtherapie}

- Anspruch und Wirklichkeit 2003

von Gerhard Rehn............................... 